\title{
Thylakoid redox signals are integrated into organellar-gene-expression-dependent retrograde signaling in the prors1-1 mutant
}

\author{
Luca Tadini ${ }^{1}$, Isidora Romani ${ }^{1}$, Mathias Pribil ${ }^{1}$, Peter Jahns ${ }^{2}$, Dario Leister ${ }^{1 *}$ and Paolo Pesaresi ${ }^{3}$ \\ ${ }^{1}$ Plant Molecular Biology (Botany), Department Biology I, Ludwig-Maximilians-Universität München, Munich, Germany \\ 2 Plant Biochemistry, Heinrich-Heine-University Düsseldorf, Düsseldorf, Germany \\ ${ }^{3}$ Dipartimento di Bioscienze, Università degli studi di Milano, Milan, Italy
}

\author{
Edited by: \\ Tatjana Kleine, \\ Ludwig-Maximilians-Universität \\ München, Germany \\ Reviewed by: \\ Shan Lu, Nanjing University, \\ China \\ Margarete Baier, FU Berlin, \\ Germany \\ ${ }^{*}$ Correspondence: \\ Dario Leister, Plant \\ Molecular Biology (Botany), \\ Department Biology I, \\ Ludwig-Maximilians-Universität \\ München, Großhaderner Str. 2, \\ D-82152 Planegg-Martinsried, \\ Germany. \\ e-mail: leister@Imu.de
}

Perturbations in organellar gene expression (OGE) and the thylakoid redox state (TRS) activate retrograde signaling pathways that adaptively modify nuclear gene expression (NGE), according to developmental and metabolic needs. The prors1-1 mutation in Arabidopsis down-regulates the expression of the nuclear gene Prolyl-tRNA Synthetase 1 (PRORS1) which acts in both plastids and mitochondria, thereby impairing protein synthesis in both organelles and triggering OGE-dependent retrograde signaling. Because the mutation also affects thylakoid electron transport, TRS-dependent signals may likewise have an impact on the changes in NGE observed in this genotype. In this study, we have investigated whether signals related to TRS are actually integrated into the OGE-dependent retrograde signaling pathway. To this end, the chaos mutation (for chlorophyll a/b binding protein harvesting-organelle specific), which shows a partial loss of PSII antennae proteins and thus a reduction in PSII light absorption capability, was introduced into the prors 1-1 mutant background. The resulting double mutant displayed a prors1-1-like reduction in plastid translation rate and a chaos-like decrease in PSII antenna size, whereas the hyper-reduction of the thylakoid electron transport chain, caused by the prors 1-1 mutation, was alleviated, as determined by monitoring chlorophyll (Chl) fluorescence and thylakoid phosphorylation. Interestingly, a substantial fraction of the nucleus-encoded photosynthesis genes down-regulated in the prors 1-1 mutant are expressed at nearly wild-type rates in prors 1-1 chaos leaves, and this recovery is reflected in the steady-state levels of their protein products in the chloroplast. We therefore conclude that signals related to photosynthetic electron transport and TRS, and indirectly to carbohydrate metabolism and energy balance, are indeed fed into the OGE-dependent retrograde pathway to modulate NGE and adjust the abundance of chloroplast proteins.

\section{INTRODUCTION}

Several features link mitochondria and plastids within the plant cell. Both organelles maintain and express genetic information, conduct electron transport functions, and are metabolically interdependent (Woodson and Chory, 2008).

In addition, the majority of mitochondrial and plastid proteins are nucleus-encoded (Kleine et al., 2009a). Therefore, to ensure that the multiprotein complexes essential for photosynthesis and respiration are assembled correctly, the activities of both organelle types must be closely coordinated with that of the nuclear genome. Signals from the organelles to the nucleus are collectively referred as retrograde signals and can largely be grouped into two categories. (1) Biogenic control, i.e., developmental control of organelle biogenesis needs to be appropriately staged and the required subunits and cofactors need to be present in correct stoichiometry for accurate assembly; and (2) operational control, representing rapid adjustments that are made to energy metabolism in response to environmental and developmental constraints to maintain optimal production and both limit and repair damage induced by oxidative stress (reviewed in Leister, 2005; Pesaresi et al., 2007; Pogson et al., 2008; Woodson and Chory, 2008; Barajas-Lopez et al., in press). Given the complexity of organellar functions, a variety of interlinked retrograde pathways can be expected (Kleine et al., 2009b; Leister, 2012).

Several metabolites have been proposed to act as messenger molecules during retrograde signaling, including (1) tetrapyrroles (Mg-protoporphyrin IX or heme) (Strand et al., 2003; Woodson et al., 2011); (2) phosphonucleotide $3^{\prime}$-phosphoadenosine 5'-phosphate (PAP) (Estavillo et al., 2011); (3) $\beta$-cyclocitral (Ramel et al., 2012); and (4) methylerythritol cyclodiphosphate (MEcPP) (Xiao et al., 2012). Moreover, plastid retrograde signaling has also been associated with responses to perturbations in photosynthetic electron transport [changes in thylakoid and stromal redox state, accumulation of the reactive oxygen species (ROS) ${ }^{1} \mathrm{O}_{2}$ and $\mathrm{H}_{2} \mathrm{O}_{2}$ (reviewed in Apel and Hirt, 2004; Oelze et al., 2008)], as well as in organellar gene expression (OGE), 
involving the pentatricopetide protein GUN1, abscisic acid (ABA) and the transcription factors PTM and ABI4 (Sullivan and Gray, 1999; Koussevitzky et al., 2007; Sun et al., 2011).

Recently, the role of Mg-protoporphyrin IX (Mg-ProtoIX) as a plastid signal has been questioned, since its accumulation following norflurazon treatment could not be correlated with changes in NGE (Mochizuki et al., 2008; Moulin et al., 2008). Consequently, it was suggested that either rapid changes in the flux through the tetrapyrrole pathway, or the accumulation of Mg-ProtoIX in a specific cellular compartment could be the origin of the plastid signal (Mochizuki et al., 2008; Moulin et al., 2008); however, these aspects deserve further investigations. Similarly, the role of ROS as classical retrograde signals is debated, because they are either probably too short-lived to reach the nucleus, as in the case of singlet oxygen ${ }^{1} \mathrm{O}_{2}$, or too unspecific $\left(\mathrm{H}_{2} \mathrm{O}_{2}\right)$ to act as information carriers (Moller and Sweetlove, 2010).

Beside the uncertainty on messenger molecules, little is also known about the extent to which different signals can be integrated into common pathways. The first insights into signaling pathways that serve to integrate chloroplast and mitochondrial activities with NGE were obtained through the characterization of mutant alleles of PRORS1 (At5g52520), an Arabidopsis gene coding for a prolyl-tRNA synthetase that is imported into both chloroplasts and mitochondria (Pesaresi et al., 2006). The leaky prors1-1 mutant allele exhibited defects in photosynthesis due to the simultaneous impairment of translation in plastids and mitochondria. Concomitantly, a specific and marked drop in the levels of transcripts of nuclear genes for proteins involved in the light reactions of photosynthesis was observed, implying that the activity of the OGE-dependent retrograde signaling pathway was altered. To investigate the specific roles of protein synthesis in mitochondria and chloroplasts in regulating nuclear photosynthetic gene expression, Arabidopsis mutants altered in mRNA translation in either mitochondria (mrpl11-1) or plastids (prpl11-1) were isolated (Pesaresi et al., 2001, 2006). Comparison of the transcript profiles of prors1-1, mrpl11-1, and prpl11-1 mutants and the double mutant mrpl11-1 prpl11-1 showed that plastids and mitochondria generate signals which act synergistically to modulate nuclear photosynthetic gene expression.

In this study, we have investigated the extent to which signals related to photosynthetic electron transport contribute to the OGE-dependent retrograde signaling pathway, by introducing the chaos mutation (Klimyuk et al., 1999) into the prors1-1 mutant background. In the chaos mutant, the $C A O$ gene (At2g47450), which codes for the chloroplast recognition particle cpSRP43, is inactivated. CpSRP43 together with cpSRP54 form the chloroplast signal recognition particle complex (Keegstra and Cline, 1999), required for the integration of the PSII antenna proteins (Lhcb proteins) into the thylakoid membranes (Schuenemann et al., 1998; Amin et al., 1999; Klimyuk et al., 1999). Consequently, chaos plants are characterized by reduced PSII antenna size, as manifested by decreased Chl $b$ and Lhcb protein contents, together with reduced levels of oxygen production and growth rate (Amin et al., 1999; Klimyuk et al., 1999). Moreover, the reduced light absorption is also associated with significantly lower foliar $\mathrm{H}_{2} \mathrm{O}_{2}$ levels than in wild type (WT), and is responsible for less photobleaching of leaves, lower induction of cytosolic ascorbate peroxidases, and lower degree of photoinhibition, indicating that chaos chloroplasts are maintained in a more oxidized state than WT (Klenell et al., 2005).

The prors1-1 chaos double mutant was compared with each single mutant in relation to rates of translation in plastids, photosynthetic performance, and NGE. The results obtained imply that signals related to photosynthetic electron transport, and indirectly to carbon metabolism and energy balance, can indeed be integrated into the OGE-dependent retrograde pathway.

\section{MATERIALS AND METHODS PLANT MATERIAL, PROPAGATION, AND GROWTH MEASUREMENTS}

The prors 1-1 mutant allele and its detection by PCR are described in Pesaresi et al. (2006). In particular, the mutation is caused by a T-DNA insertion ( $p A C 106)$ at $-44 \mathrm{bp}$ from the translation starting codon. The gene-specific primers prors1-sense (5'-AACCAAGCATGAGTTTCTCG-3') and prors1-antisense $\left(5^{\prime}\right.$-ATCCGGAAAGAGGTCTGTTC- $\left.3^{\prime}\right)$ were employed to detect the WT PRORS1 allele; the T-DNA-specific primer T9697 (5'-CTCTTTCTTTTTCTCCATATTGACCAT-3') and prors1-antisense were used to identify the prors 1-1 mutant allele. The chaos mutant used in this study was identified in a population mutagenized with the En transposon (Wisman et al., 1998) based on its photosynthetic performance and leaf pigment composition (Varotto et al., 2000). The mutant allele carries an En insertion (which is stable because of 249-bp deletion at the left border) at position +149 (relative to the start codon) in the single-exon gene CAO. The gene-specific primers cao-sense (5'-ATGCAAAAGGTCTTCTTGGC- $\left.3^{\prime}\right)$ and cao-antisense (5'-CCTCTCTCGTCTTCCACTTC- $\left.3^{\prime}\right) \quad$ were employed in PCRs to detect the WT CAO allele; the En-specific primer EnR (5'-GAGCGTCGGTCCCCACACTTCTATAC-3') and cao-antisense were used to identify the chaos mutant allele. The prors 1-1 chaos double mutant was obtained by crossing prors1-1 and chaos single mutants and PCR-genotyping F2 individuals. Arabidopsis thaliana Heynh. WT (Col-0) and mutant plants were grown under controlled growth chamber conditions as described (Pesaresi et al., 2009). The method used for growth measurement has been described before (Leister et al., 1999).

\section{NUCLEIC ACID ANALYSIS}

A. thaliana DNA was isolated as described (Ihnatowicz et al., 2004). For RNA analysis, total leaf RNA was extracted from fresh tissue using the TRIzol reagent (Invitrogen, Germany). Northern analysis was performed under stringent conditions, according to Sambrook and Russell (2001). Probes complementary to nuclear or chloroplast genes were used for the hybridization experiments. Primers used to amplify the probes are listed in Table $\mathbf{1}$. All probes used were cDNA fragments labeled with ${ }^{32} \mathrm{P}$. Signals were quantified with a phosphoimager (Typhoon; GE Healthcare, Munich, Germany) using the program ImageQuant (version 1.2; Molecular Dynamics). For quantitative real-time PCR (qRTPCR) analysis, $4 \mu \mathrm{g}$ aliquots of total RNA, treated with DNase I (Roche Applied Science) for at least $30 \mathrm{~min}$, were utilized for first-strand cDNA synthesis using iScript reverse transcriptase 
Table 1 | Oligonucleotide sequences employed for gene expression analysis.

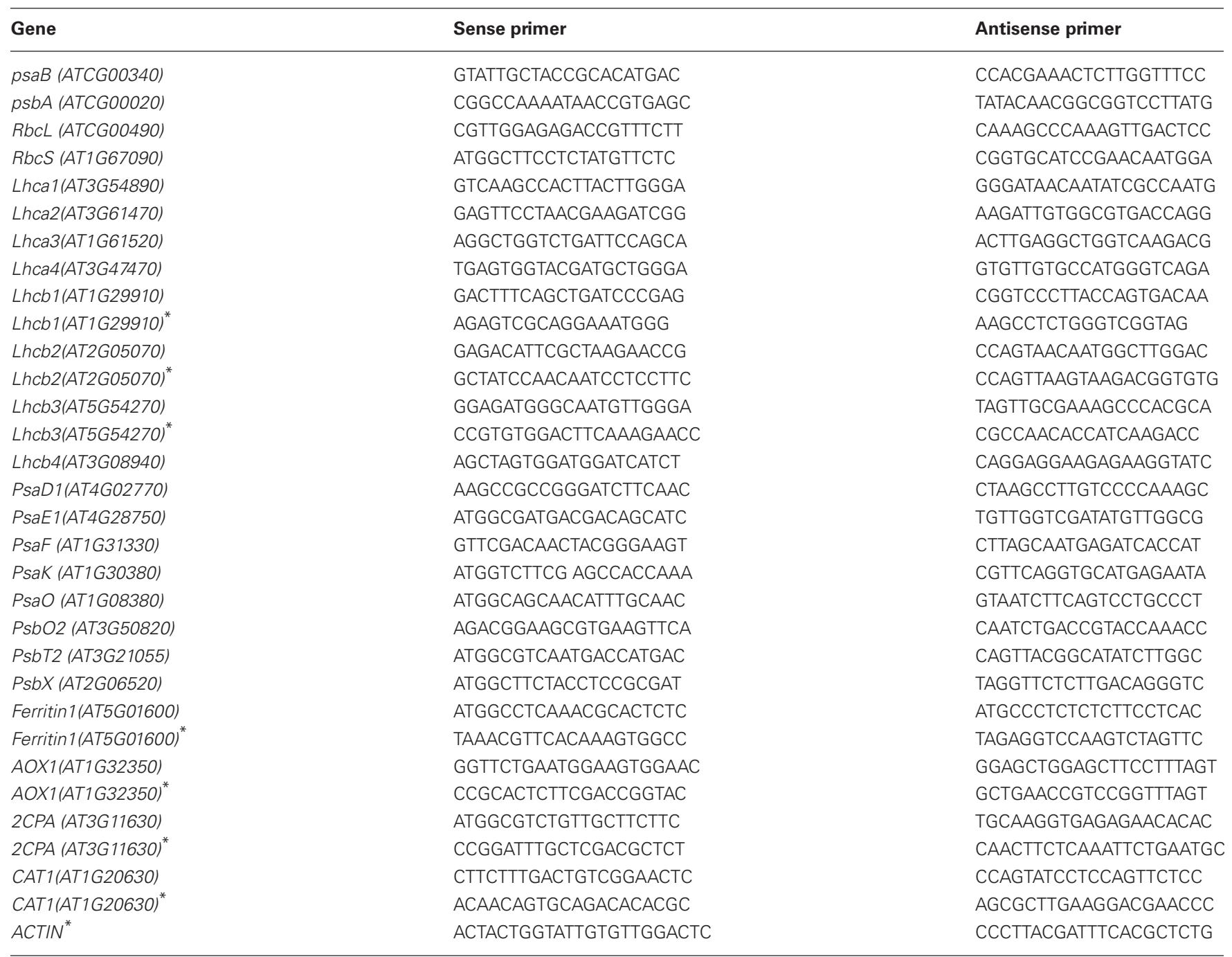

Asterisk indicates primer pairs employed for qRT-PCR analyses.

(Bio-Rad) according to the supplier's instructions. The qRT-PCR profiling was carried out on an iCycler iQ5 real-time PCR system (Bio-Rad), using the oligonucleotide sequences reported in Table 1. Actin was used as internal standard. Data from three biological and three technical replicates were analyzed with Bio-Rad iQ5 software (version 2.0).

\section{PAGE AND IMMUNOBLOT ANALYSES}

Leaves were harvested from plants at the 6-leaf rosette stage, and thylakoids were prepared as described (Bassi et al., 1985). For SDS-PAGE, thylakoid proteins isolated from equal amounts of leaf material (fresh weight) were fractionated on denaturing Trisglycine SDS-PAGE gels (with 12\% PA) and the protein content was stained with colloidal Coomassie blue (G 250).

For immunoblot analyses, total proteins were prepared from plants at the 6-leaf rosette stage (Martinez-Garcia et al., 1999), then fractionated by SDS-PAGE (on 12\% polyacrylamide gels) (Schägger and von Jagow, 1987). Subsequently, proteins were transferred to poly(vinylidene difluoride) membranes (Ihnatowicz et al., 2004), and replicate filters were probed with appropriate antibodies. Signals were detected by enhanced chemiluminescence (GE Healthcare). Thylakoid protein phosphorylation was monitored with a phosphothreonine-specific antibody (Cell Signaling Technology) in total leaf protein extracts obtained from WT and mutant plants kept overnight in the dark and then exposed to light $\left(80 \mu \mathrm{mol}\right.$ photons $\left.\mathrm{m}^{-2} \mathrm{~s}^{-1}\right)$ for $4 \mathrm{~h}$.

Coomassie-stained gels and immunoblots were scanned and quantified using ImageQuant (version 1.2; Molecular Dynamics).

\section{In vivo TRANSLATION ASSAY}

The in vivo translation assay was performed essentially as in Pesaresi (2011). Twelve leaf discs (4 cm in diameter) were incubated in a buffer containing $20 \mu \mathrm{g} / \mathrm{ml}$ cycloheximide, $1 \mathrm{mM}$ $\mathrm{K}_{2} \mathrm{HPO}_{4}-\mathrm{KH}_{2} \mathrm{PO}_{4}(\mathrm{pH} 6.3$ ), and $0.1 \%(\mathrm{w} / \mathrm{v})$ Tween-20 to block cytosolic translation. $\left[{ }^{35} \mathrm{~S}\right]$ methionine was added to the buffer $(0.1 \mathrm{mCi} / \mathrm{ml})$ and infiltrated into the discs under vacuum. Leaves 
were exposed to light $\left(20 \mu \mathrm{mol}\right.$ photons $\left.\mathrm{m}^{-2} \mathrm{~s}^{-1}\right)$ and four leaf discs were collected at each time point $(5,15$, and $30 \mathrm{~min})$. Total proteins were extracted as described above and fractionated by Tris-glycine SDS-PAGE (12\% PA). Signals were detected and quantified using the phosphoimager and the ImageQuant program as described above.

\section{Chl FLUORESCENCE, OXYGEN EVOLUTION, AND PIGMENT ANALYSES}

In vivo $\mathrm{Chl}$ a fluorescence of leaves was measured using the Dual-PAM-100 (Walz, Effeltrich, Germany) as described (Pesaresi et al., 2009). Five plants of each genotype were analyzed and average values plus standard deviations were calculated. Plants were first dark-adapted for $30 \mathrm{~min}$ and minimal fluorescence $\left(F_{0}\right)$ was measured. Then pulses $(0.8 \mathrm{~s})$ of saturating white light $\left(5000 \mu \mathrm{mol}\right.$ photons $\mathrm{m}^{-2} \mathrm{~s}^{-1}$ ) were used to determine the maximum fluorescence $\left(F_{M}\right)$, and the ratio $\left(F_{M}-F_{0}\right) / F_{M}=F_{V} / F_{M}$ (maximum quantum yield of PSII) was calculated. An 8-min exposure to actinic red light $\left(37 \mu \mathrm{mol}\right.$ photons $\left.\mathrm{m}^{-2} \mathrm{~s}^{-1}\right)$ served to drive electron transport between PSII and PSI at steady state. In particular, the employed routine allowed to measure the steady-state fluorescence $\left(F_{S}\right)$ and the maximum fluorescence after light adaptation $\left(F_{M^{\prime}}\right)$ (saturation pulse, $0.8 \mathrm{~s}, 5000 \mu \mathrm{mol}$ photons $\left.\mathrm{m}^{-2} \mathrm{~s}^{-1}\right)$ every $20 \mathrm{~s}$. The ratio $\left(F_{M^{\prime}}-F_{S}\right) / F_{M^{\prime}}$ gives the effective quantum yield of PSII $\left(\Phi_{\mathrm{II}}\right)$, while the excitation pressure parameter 1-qL reflects the size of the reduced fraction of $\mathrm{Q}_{\mathrm{A}}$. The coefficient of photochemical quenching, $\mathrm{qL}$, was calculated as $\left(F_{M^{\prime}}-F_{S}\right) /\left(F_{M^{\prime}}-F_{0^{\prime}}\right) \times F_{0^{\prime}} / F_{S}$ (Kramer et al., 2004), with $F_{0^{\prime}}$ being the minimum fluorescence after removal of the illumination. A 2-min dark period was also employed to monitor the recovery to the maximum quantum yield of PSII.

In vivo $\mathrm{Chl}$ a fluorescence of whole plants was recorded using an imaging Chl fluorometer (Imaging PAM; Walz, Germany). Dark-adapted plants were exposed to a pulsed, blue measuring beam $\left(1 \mathrm{~Hz}\right.$, intensity $\left.4 ; F_{0}\right)$ and a saturating light flash (intensity 4$)$ to obtain $F_{V} / F_{M}$. A 10 -min exposure to actinic light $\left(80 \mu \mathrm{mol}\right.$ photons $\left.\mathrm{m}^{-2} \mathrm{~s}^{-1}\right)$ was then used to calculate $\Phi_{\mathrm{II}}$ at the steady state.

Rates of oxygen evolution from leaf discs were measured with a Clark-type oxygen electrode (Hansatech Instruments Ltd.) as described by Havaux and Devaud (1994). In particular, measurements were performed at saturating $\mathrm{CO}_{2}$ concentration by employing a bicarbonate/carbonate buffer. The light limited rates of photosynthetic $\mathrm{O}_{2}$ evolution were measured at $80 \mu \mathrm{mol}$ photons $\mathrm{m}^{-2} \mathrm{~s}^{-1}$. Pigments were analyzed by reversed-phase HPLC (Farber et al., 1997).

\section{RESULTS}

\section{LIGHT ABSORPTION AND OXYGEN EVOLUTION ARE REDUCED IN prors1-1 chaos LEAVES}

The double mutant prors1-1 chaos was generated as described in "Materials and Methods." Like the corresponding single mutants prors1-1 and chaos, the prors1-1 chaos double mutant has palegreen cotyledons and leaves, and is smaller than WT plants of the same age (Figure 1A). Quantification of growth rates by noninvasive image analysis under growth chamber conditions showed that while prors $1-1$ and chaos mutants were about 20 and 30\% smaller, respectively, than WT plants at 4 weeks of age, the prors1-1 chaos double mutant displayed a size reduction of more than $50 \%$ relative to WT plants (Figures $\mathbf{1 A}, \mathbf{B}$ ). In addition, reductions in petiole length could be observed in prors1-1, chaos and, more markedly, in prors $1-1$ chaos mutants during the first stages of plant development, whereas such differences were less pronounced in adult plants (Figure 1C). Col-0, prors1-1, and chaos plants showed also a very similar number of leaves throughout the life cycle, whereas the leaf number was slightly decreased in prors 1-1 chaos plants, particularly during the first 2 weeks after germination (Figure 1D).

Four-week-old WT and mutant plants were also subjected to $\mathrm{Chl}$ a fluorescence measurements to monitor photosynthetic performance (Figure 2). The data showed a lower maximum quantum yield of PSII $\left(F_{V} / F_{M}\right)$ in prors1-1, whereas values higher than in WT were observed in chaos leaves. Accordingly, the chaos mutation was able to restore $F_{V} / F_{M}$ to the WT level in prors1-1 chaos leaves (Figures 2A,C). When the $\Phi_{\text {II }}$ parameter, reflecting the effective quantum yield of PSII, was taken into account, it could be also observed that prors1-1 plants showed reduced $\Phi_{\text {II }}$ values with respect to Col-0, whereas chaos and prors1-1 chaos values were even higher than those of WT leaves (Figures 2B,C). In agreement with these observations, a decrease in the degree of reduction of $\mathrm{Q}_{\mathrm{A}}$ (the primary electron acceptor of PSII) and the plastoquinone (PQ) pool (measured as 1-qL; a parameter frequently used for an indirect estimation of the redox state of the PQ pool; Kramer et al., 2004; Jung et al., 2010) was observed in chaos and prors1-1 chaos leaves (Figure 2D), suggesting a reduced net electron injection into the thylakoid transport chain, caused by chaos mutation. This aspect was investigated further by measuring oxygen production with a Clark-type oxygen electrode under standard lighting conditions. The levels of oxygen production per unit of leaf area were always found to be lower in chaos and prors1-1 chaos leaves than in Col- 0 or the prors $1-1$ single mutant (chaos, $0.014 \pm 0.002 \mu \mathrm{mol}$ $\mathrm{m}^{-2} \mathrm{~h}^{-1}$; prors $1-1$ chaos, $0.011 \pm 0.003$; prors $1-1,0.023 \pm 0.004$ Col-0, $0.029 \pm 0.003)$.

To quantify the alteration in leaf coloration in prors 1-1, chaos, and prors1-1 chaos plants, leaf pigments were analyzed by HPLC (Table 2). As expected, the total $\mathrm{Chl}$ content $(\mathrm{Chl} \mathrm{a}+\mathrm{b})$ was reduced by about 57 and $66 \%$ in chaos and prors $1-1$ chaos leaves, respectively, and the $\mathrm{Chl} \mathrm{a} / \mathrm{b}$ ratio was 3.69 in chaos and 3.01 in prors1-1 chaos leaves, with respect to 2.71 in Col-0. In addition, carotenoids that bind specifically to PSII antenna proteins (Lhcb), such as neoxanthin (Nx), lutein (Lut), and the VAZ pool (violaxanthin + antheraxanthin + zeaxanthin) were markedly reduced in chaos and prors 1-1 chaos mutants. In contrast, $\beta$-carotene, which associates with PSII-core proteins, showed only a marginal decrease in mutant leaves, confirming the specific decrease in levels of PSII antenna proteins in the mutants containing the chaos allele.

Taken together, the data indicate that, in the prors1-1 chaos double mutant, the reduction in the size of the PSII antenna caused by the chaos mutation, and the resulting decrease in the total amount of light absorbed, effectively counteracts the increase in thylakoid electron pressure due to prors1-1. Hence, the excessive reduction of the thylakoid electron transport chain 
A
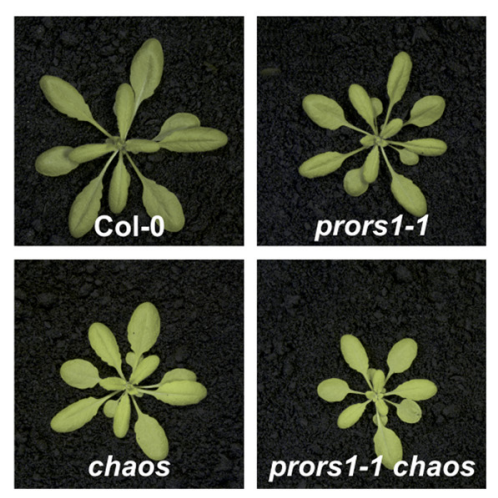

C

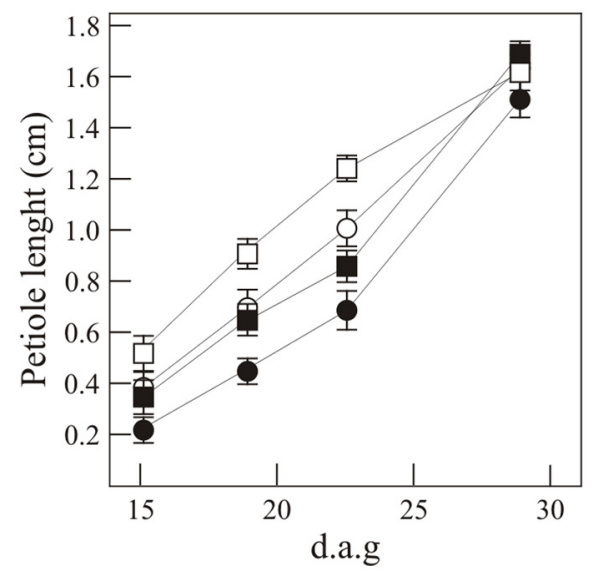

FIGURE 1 | Phenotypes of mutant (prors 1-1, chaos, and prors 1-1 chaos) and WT (Col-0) plants. (A) The different genotypes were grown for 4 weeks in the growth chamber. (B) The growth rates of the different genotypes were measured from $4 \mathrm{~d}$ to $28 \mathrm{~d}$ after germination (d.a.g.).

(C) Petiole length was measured by monitoring the petiole of the fifth true
$\mathbf{B}$

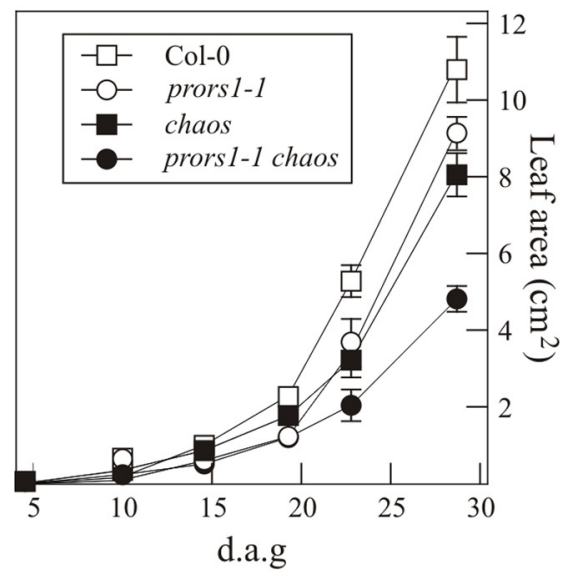

D

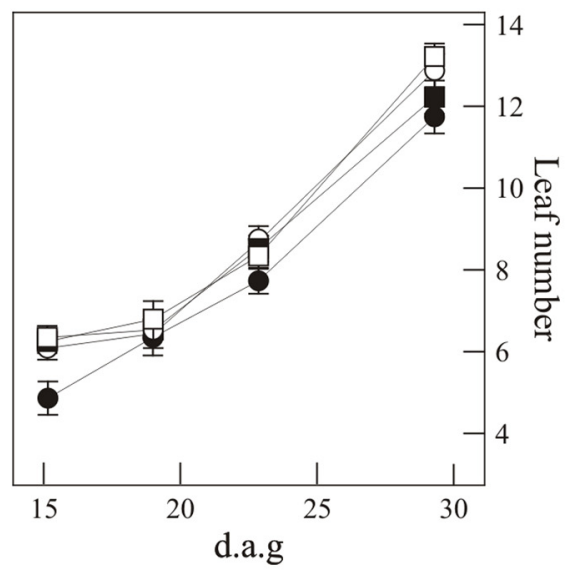

leaf from 15 to 28 d.a.g. (D) The number of true leaves was determined during the period from 15 to 28 d.a.g. Each point reported in (B), (C), and (D) is based on the determination of average leaf area, petiole length and leaf number, respectively, in at least 12 individuals. Bars indicate standard deviations. revealed by the increased $1-\mathrm{qL}$ values in the prors $1-1$ mutant is alleviated in prors1-1 chaos plants.

\section{LEVELS OF LIGHT-HARVESTING COMPLEXES AND THYLAKOID PHOSPHORYLATION ARE REDUCED IN prors1-1 chaos LEAVES}

To determine the effects of the combined action of the chaos and prors 1-1 mutations on thylakoid protein composition, membranes isolated from WT and mutant plants were fractionated by 1D SDS-PAGE (Figure 3A). As expected, densitometric analyses revealed a decrease of about $70 \%$ for the major lightharvesting complex of PSII (Lhcb1, Lhcb2, Lhcb3) in both chaos and prors 1-1 chaos plants (Table 3). In addition, prors 1-1 and prors 1-1 chaos thylakoids were characterized by a decrease in the $\alpha$ - and $\beta$-subunits of the ATPase complex by 47 and $56 \%$, respectively. A more detailed picture of thylakoid protein composition in WT and mutant plants was obtained through immunoblot analysis (Figure 3B, Table 3). In particular, some PSII antenna proteins, such as Lhcb1, Lhcb2, and Lhcb6, showed similar reductions in both chaos and prors1-1 chaos thylakoids, whereas Lhca1 accumulation in prors1-1 chaos appeared to be the result of an additive effect of the two single mutations. Levels of most of the other subunits analyzed, including polypeptides from the PSI antenna (Lhca2), the PSII antenna (Lhcb5), the cores of PSI (PsaD and PsaF) and PSII (D1, CP43, and D2), and the oxygen-evolving complex (PsbQ), were higher (i.e., closer to WT-like levels) in prors1-1 chaos than in prors1-1 thylakoids. No marked differences were observed between WT and mutant plants with respect to the accumulation of PsbO (a subunit of the oxygen-evolving complex), PsbS (subunit of PSII involved in non-photochemical quenching of $\mathrm{Chl}$ fluorescence) or the large subunit of RubisCO (RbcL).

The major decrease in PSII antenna proteins and the lower 1-qL values observed in chaos and prors1-1 chaos leaves imply that the thylakoid electron transport chain is more oxidized in these genotypes than in prors 1-1 plants. Because the level of thylakoid protein phosphorylation is directly linked to the reduction state of the PQ pool, immunoblot analysis using a phosphothreonine-specific antibody was carried out 
A
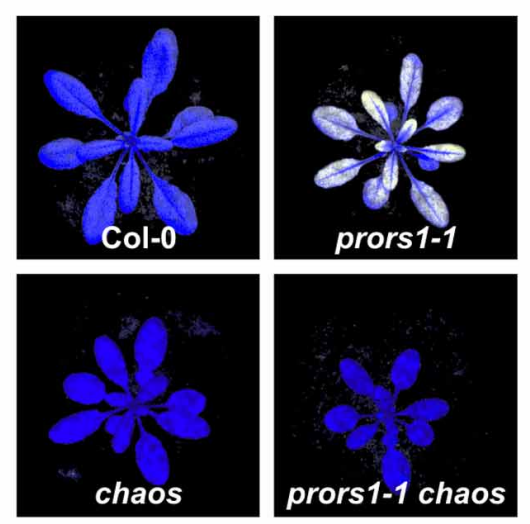

B
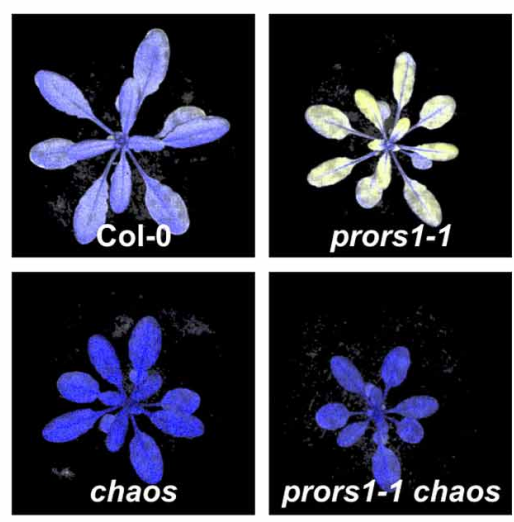

C

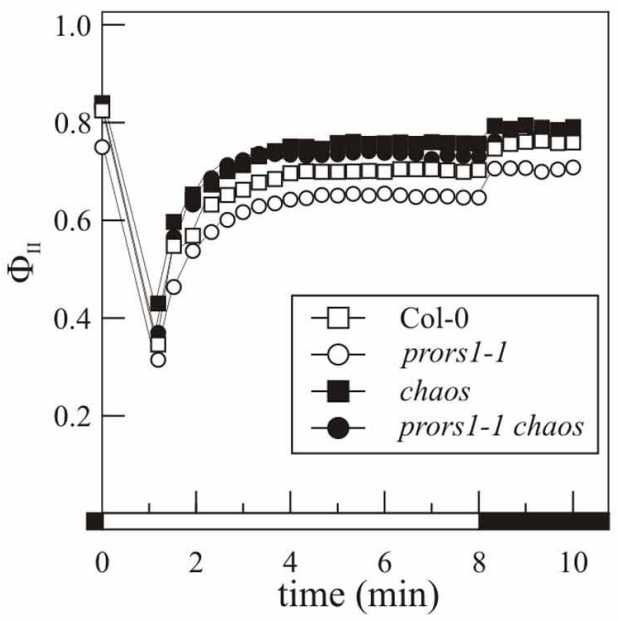

FIGURE 2 | Photosynthetic performance of mutant (prors 1-1, chaos, and prors 1-1 chaos) and WT (Col-0) plants. The photosynthetic parameters $F_{V} / F_{M}$ (A) and $\Phi_{\|}$(B) were measured in the different genotypes, by employing an imaging $\mathrm{Chl}$ fluorometer, as described in "Materials and Methods." Signal intensities for $F_{V} / F_{M}$ and $\Phi_{\|}$are
D

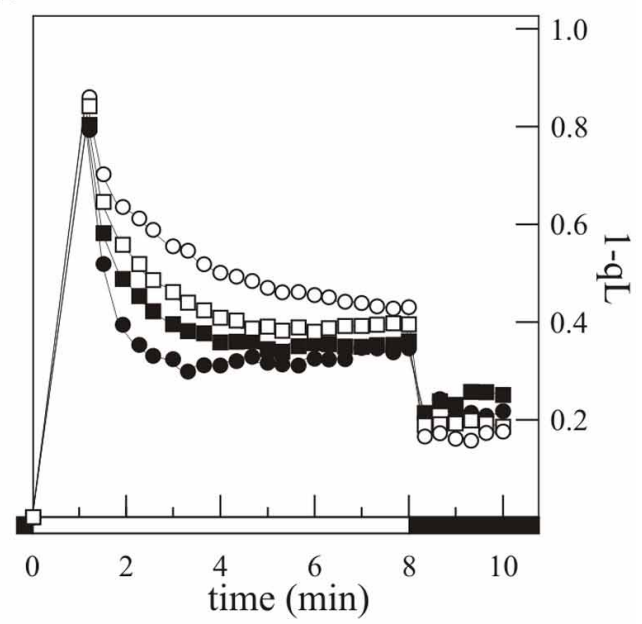

indicated according to the color scale at the bottom of the panel. $F_{V} / F_{M}$ (time 0), $\Phi_{\|}$(C) and 1-qL (D) were also measured using the Dual-PAM-100, as described in "Materials and Methods." Black bars indicate dark periods, and white bars light periods. Standard deviations were always below $10 \%$.

Table 2 | Levels of leaf pigments in light-adapted mutant (prors1-1, chaos, and prors 1-1 chaos) and WT (Col-0) plants at the 6-leaf rosette stage.

\begin{tabular}{|c|c|c|c|c|c|c|c|c|}
\hline & \multicolumn{8}{|c|}{ Leaf pigment content (pmol/mg leaf) } \\
\hline prors1-1 & $45 \pm 3$ & $154 \pm 10$ & $480 \pm 28$ & $1225 \pm 71$ & $121 \pm 8$ & $66 \pm 1$ & $1705 \pm 68$ & $2.55 \pm 0.15$ \\
\hline chaos & $29 \pm 1$ & $103 \pm 9$ & $197 \pm 6$ & $728 \pm 34$ & $176 \pm 7$ & $34 \pm 3$ & $925 \pm 40$ & $3.69 \pm 0.08$ \\
\hline prors 1 -1 chaos & $24 \pm 3$ & $92 \pm 9$ & $165 \pm 12$ & $497 \pm 39$ & $174 \pm 10$ & $43 \pm 4$ & $742 \pm 61$ & $3.01 \pm 0.09$ \\
\hline
\end{tabular}

Leaf pigments were quantified by HPLC and are reported in pmol/mg leaf (fresh weight). Mean values \pm SD are shown. Nx, neoxanthin; Lut, lutein; ChI b, chlorophyll b; Chl a, chlorophyll a; $\beta$-Car, $\beta$-carotene; VAZ, violaxanthin + antheraxanthin + zeaxanthin.

to investigate this further (Figure 3C). PSII-core proteins and LHCII phosphorylation were markedly increased in lightadapted prors 1-1 thylakoids, in particular when the reduced accumulation of these proteins in prors1-1 plants (Table 3) is accounted for. On the other hand, a marked decrease of D1, D2, and CP43 phosphorylation was observed in lightadapted thylakoids isolated from chaos and prors1-1 chaos leaves. In the dark, only marginal levels of thylakoid protein 

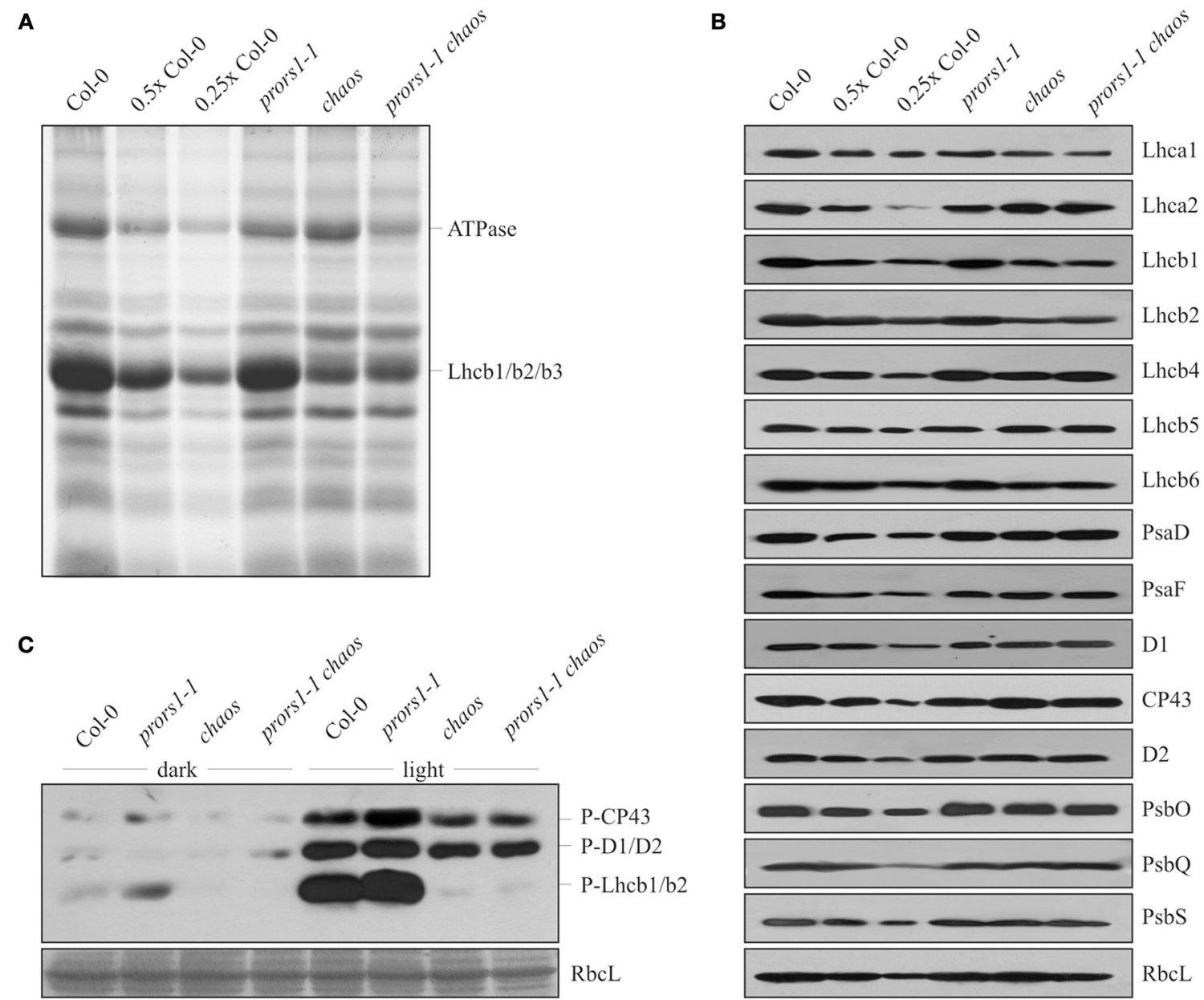

FIGURE 3 | 1D SDS-PAGE and immunoblot analysis of thylakoid proteins from WT (Col-0) and mutant (prors 1-1, chaos, and prors 1-1 chaos) leaves. (A) Thylakoid proteins isolated from equal amounts of leaf (fresh weight) were fractionated by SDS-PAGE and stained with Coomassie Brilliant blue. Band intensities were quantified by densitometric analyses (see "Materials and Methods"). (B) Identical amounts of total leaf proteins were fractionated by SDS-PAGEs as in (A), transferred onto nylon filters and probed with antibodies raised against individual subunits of PSI (PsaD, PsaF), PSII (D1, CP43, D2, PsbO, PsbQ,
PsbS), LHCl (Lhca1, Lhca2) and LHCII (Lhcb1, Lhcb2, Lhcb4, Lhcb5, Lhcb6), and the large subunit of RubisCO (RbcL). The lanes marked $0.5 x$ Col-0 and $0.25 \times$ Col- 0 were loaded with the indicated dilutions of the standard control sample. (C) Identical amounts of total proteins isolated from dark- and light-adapted plants were fractionated by SDS-PAGE as in (A), blotted onto nylon filters and probed with a phosphothreonine (P-Thr)-specific antibody. A portion of the Coomassie Brilliant Blue-stained gel, corresponding to the RbcL migration region, was used to check for equal loading. phosphorylation were detected in the different genetic backgrounds.

Thus, the polypeptide composition of prors1-1 chaos thylakoids seems to be influenced by two major factors. Additive effects of the two single mutations prors 1-1 and chaos seem to act on the abundance of Lhca1, Lhcb2, and the ATPase complex, while another group of photosynthetic proteins, the abundance of which is unaffected in the chaos mutant but decreased in prors 1 1(e.g., D1, PsaF, PsbQ, Lhca2, Lhcb4, and Lhcb5), behaves in a WT-like manner in prors 1-1 chaos plants. These findings indicate (1) that the increased excitation pressure in prors1-1 thylakoids (revealed by the increase in the 1-qL value and in thylakoid phosphorylation) markedly affects the accumulation of certain photosynthetic proteins, and (2) that this effect can be attenuated by reducing PSII antenna size through the chaos mutation.

\section{PLASTID PROTEIN SYNTHESIS IS UNAFFECTED BY THE chaos MUTATION}

To determine whether the differences in thylakoid protein accumulation observed between prors1-1 and prors1-1 chaos plants were due to chaos-dependent adaptive mechanisms that can modulate the translation process, plastid protein synthesis was investigated. To this end, the rate of incorporation of $\left[{ }^{35} \mathrm{~S}\right]$ methionine into plastid proteins in young leaves of WT and mutant (prors 1-1, chaos and prors1-1 chaos) plants was monitored for 5, 15, and $30 \mathrm{~min}$ in the presence of light and inhibitors of cytoplasmic protein synthesis (Figure 4). Subsequently, total leaf proteins were extracted and fractionated by SDS-PAGE. In five independent experiments, the amounts of RbcL labeled in prors1-1 and prors1-1 chaos plants were comparable, and equivalent to about $55 \%$ of WT levels. A marginal decrease in PSII-D1 signals was 
Table 3 | Quantification of thylakoid (phospho)proteins in light-adapted mutant (prors1-1, chaos, and prors1-1 chaos) and WT (Col-0) plants.

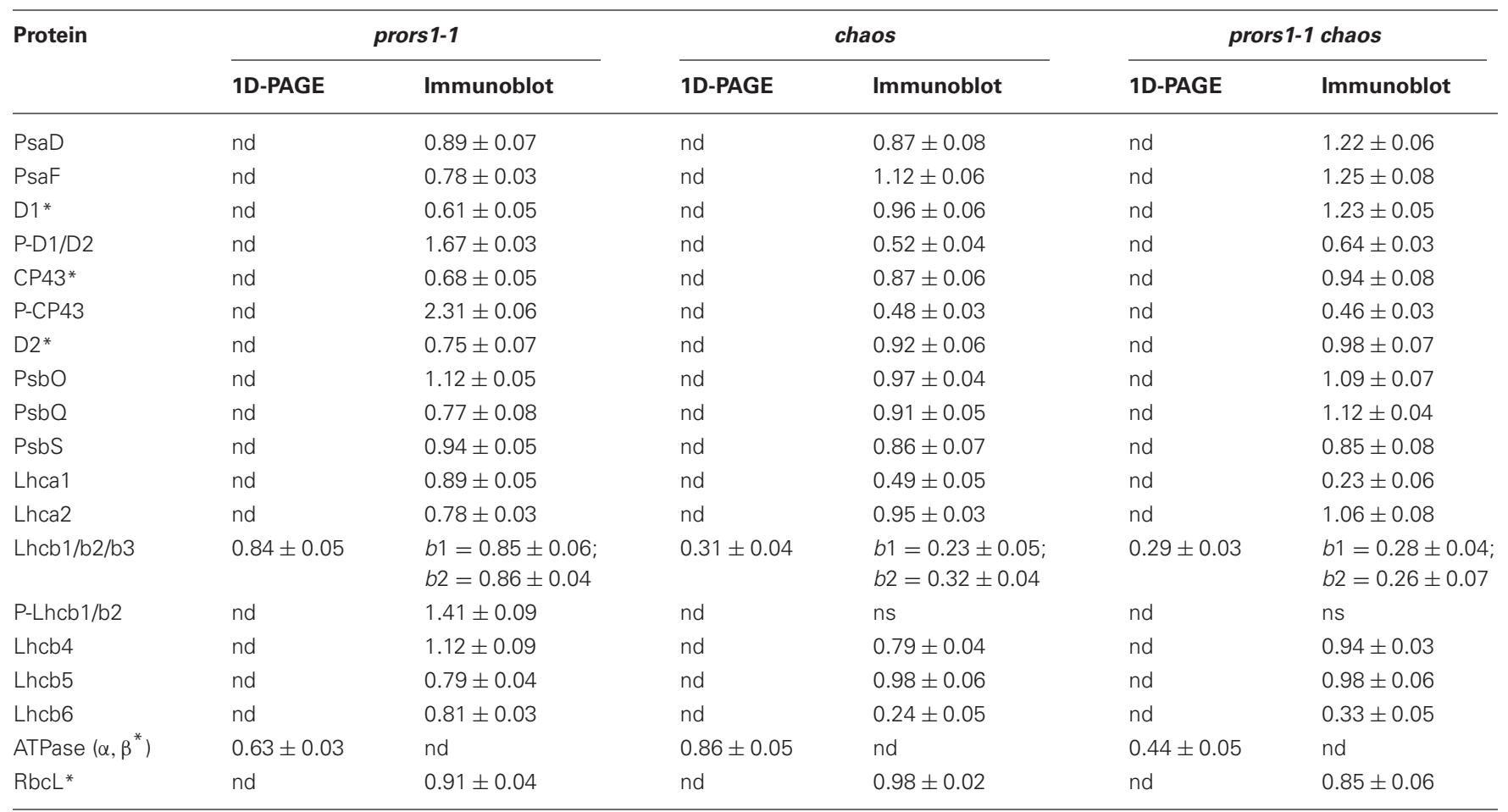

WT levels are set to $100 \%$. Average values were calculated from three independent 1D-PA gels and protein gel blots (see Figure 3). nd, not determined; ns, not significant.

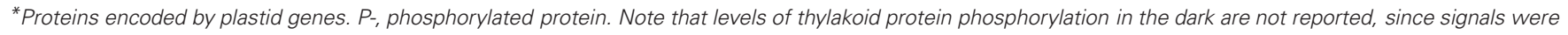
barely detectable.

also observed in these two mutants, whereas chaos plants behaved essentially like WT in this respect.

Taken together, these results exclude the possibility that the differences in thylakoid protein accumulation observed in prors1-1 and prors 1-1 chaos mutants result from differences in the rate of plastid protein synthesis. Therefore, the increased accumulation of plastid-encoded subunits in prors 1-1 chaos (relative to prors1-1) leaves must be attributed to differences in post-translational events (including greater protein stability) associated with the lower level of oxidative damage incurred when light absorption and photosynthetic electron transport are less efficient. The nearly WT abundance of nucleus-encoded proteins observed in prors1-1 chaos thylakoids might be due to increases in transcription, translation, or protein stability, or any combination of these.

\section{THE chaos MUTATION PARTIALLY SUPPRESSES THE OGE-DEPENDENT RETROGRADE SIGNALING PATHWAY}

To assess the relative contribution of photosynthetic electron transport and the associated changes in TRS to the OGEdependent signaling pathway, expression analyses of plastid and nuclear photosynthesis genes were conducted on WT and mutant plants. Probes for one plastid and nine nuclear genes encoding subunits of PSI and its antenna, one plastid and seven nuclear genes encoding subunits of PSII and its antenna, and for the plastid-encoded large subunit (RbcL) and the nucleus-encoded small subunit ( $\mathrm{RbcS}$ ) of RubisCO were hybridized to RNA gel blots loaded with total RNA from lightadapted leaves (Figure 5A). As expected, all nuclear photosynthesis genes were down-regulated in prors1-1 plants, confirming the role of mitochondrial and plastid translation rate in triggering photosynthesis-related NGE (Figure 5A). However, in the prors 1-1 chaos double mutant, 15 of the 17 nuclear photosynthesis genes analyzed were up-regulated relative to prors1-1. In particular, expression of Lhca1, Lhca2, PsaE1, PsaF, PsaK, PsaO, Lhcb1, Lhcb2, and PsbX in prors1-1 chaos leaves was identical to (and in the case of PsbT2 even higher than) that in WT. The remaining genes ( $P s a D 1, L h c b 3, L h c b 4, P s b O 2$, and $R b c S)$ were derepressed in prors1-1 chaos, in some cases to levels similar to those seen in the chaos single mutant, but lower than in WT. Exceptions were represented by Lhca3 and Lhca4 genes, which were down-regulated in both prors1-1 and prors1-1 chaos mutants. The limited capacity for light absorption caused by the chaos mutation also influences plastid gene expression, as shown by the marked drop in $p s a A-B$ expression in chaos and prors $1-1$ chaos mutants, whereas $p s b A$ and $R b c L$ levels were almost unchanged in mutant plants.

Expression of genes involved in scavenging or preventing the formation of ROS was also investigated. The levels of transcripts of Ferritin1, mitochondrial alternative oxidase (AOX1), catalase (CAT1) and 2-Cys-peroxiredoxin-A (2CPA), expression of which has been reported to be stimulated by increases in ROS production, were only slightly altered in the mutant genotypes. 


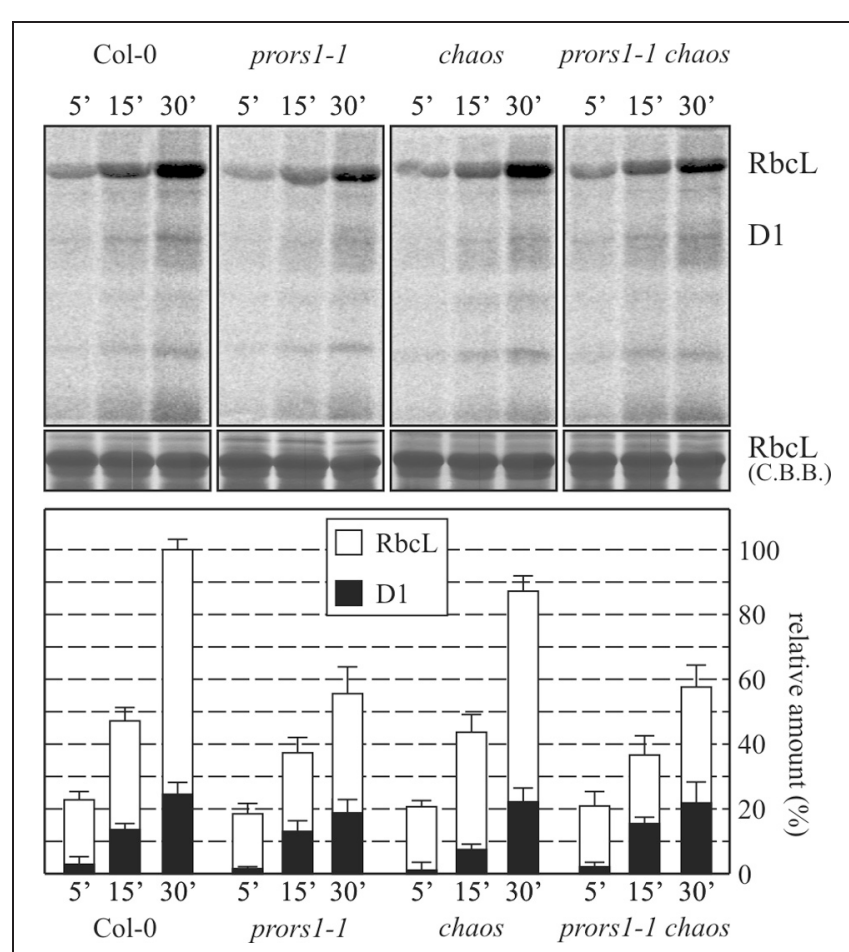

FIGURE 4 | Translational efficiency of chloroplast-encoded mRNAs in WT (Col-0) and mutant (prors 1-1, chaos, and prors 1-1 chaos) leaves. Leaves isolated from 6-leaf-rosette plants were pulse-labeled with $\left.{ }^{35} \mathrm{~S}\right]$ methionine under low-level illumination $\left(20 \mu \mathrm{mol}\right.$ photons $\left.\mathrm{m}^{-2} \mathrm{~s}^{-1}\right)$ for 5,15 , and $30 \mathrm{~min}$ in the presence of cycloheximide to inhibit cytosolic protein synthesis. Total leaf proteins were then isolated, fractionated by SDS-PAGE, and detected by autoradiography. A portion of the Coomassie Brilliant Blue (CBB)-stained gel, corresponding to the RbcL migration region, was employed as internal standard for data normalization. Levels of ${ }^{35}$ S]methionine incorporation into RbcL and D1 proteins were quantified and are shown in the histogram. Values are averages of 5 independent experiments and were normalized to the maximal signal intensities obtained in WT leaves after 30 min labeling.

In order to validate the gene expression data obtained by Northern blot hybridizations, the expression of Lhcb1, Lhcb2, Lhcb3, Ferritin1, AOX1, 2CPA, and CAT1, all of them belonging to large gene families, was also monitored by qRT-PCR analyses (Figure 5B). In this case too, $L h c b$ expression was specifically down-regulated in prors1-1 leaves and derepressed in prors1-1 chaos plants. On the contrary, the expression of all the other genes remained unchanged between Col-0 and mutant leaves.

Taken together, the data clearly demonstrate that the OGEdependent signaling pathway is tightly linked to photosynthetic electron transport and the associated TRS. In the prors1-1 chaos double mutant, the significant reduction in light absorption and oxygen evolution caused by the chaos mutation largely prevents the specific down-regulation of nuclear photosynthetic genes caused by the prors1-1 mutation. Interestingly, this effect is likely to be caused by the altered redox state of the thylakoid electron transport chain (as shown by reduced levels of thylakoid protein phosphorylation and $p s a A-B$ operon expression), not by a burst of ROS production, as indicated by the limited changes in the expression of ROS-induced genes.

\section{DISCUSSION}

Attempts to distinguish between different retrograde signaling pathways have been hampered by difficulties in discriminating between primary and secondary effects caused by chemical inhibitors, and a comparative lack of genetic mutants that influence organellar function in a specific, well-defined manner (Leister, 2012; Barajas-Lopez et al., in press). In this study we have investigated the retrograde signaling pathway(s) active in prors 1-1 mutant plants, which exhibit perturbations in two chloroplast functions relevant for retrograde signaling: (1) a mild defect in protein synthesis in both mitochondria and chloroplasts; and (2) altered photosynthetic electron transport (this manuscript; Pesaresi et al., 2006).

\section{DIMINISHED LIGHT ABSORPTION MITIGATES THE DEFECT IN PHOTOSYNTHETIC ELECTRON TRANSPORT IN prors1-1 PLANTS}

The excessively reduced state of the thylakoid electron transport chain observed in the prors $1-1$ mutant, as indicated by increased thylakoid protein phosphorylation, reduced effective quantum yield of PSII, and increased 1-qL values, supports the notion that many alterations in plant cell metabolism directly or indirectly impinge on the redox state of photosynthetic electron transport components, making the photosynthetic apparatus a major sensor of physiological imbalances (Pfannschmidt and Yang, 2012). Therefore, changes in thylakoid excitation pressure may be associated with major modifications in gene expression at the organellar and nuclear levels. Here, we have specifically addressed this issue by introducing the chaos mutation into prors 1-1 plants. This mutation in the CAO gene reduces the size of the PSII antenna, thus mimicking a major adaptive mechanism that plants have evolved to protect themselves against the damaging effects of excess light energy (for a review see: Oelze et al., 2008). Downregulation of the $C A O$ gene, which codes for the cpSRP43 subunit involved in the insertion of Lhcb proteins into the thylakoids (Klimyuk et al., 1999), has actually been implicated in the system that remodels the photosynthetic machinery to safeguard against photo-oxidative stress (Klenell et al., 2005). And indeed, in prors1-1 chaos double mutant plants, the thylakoid electron transport chain is more highly oxidized, as indicated by the higher $\Phi_{\mathrm{II}}$ and lower 1-qL values, as well as reduced $\mathrm{O}_{2}$ production relative to prors $1-1$ plants, and the concomitant reduction in thylakoid protein phosphorylation. These data support previous findings where chaos seedlings have been reported to be highly tolerant to photooxidative stress under both tightly controlled laboratory conditions and highly variable conditions in the field, and to mitigate the effects of the lesion simulating disease 1 ( $l s d 1$ ) mutation (Mateo et al., 2004; Klenell et al., 2005). These effects are attributable to a decrease in light absorption resulting from the limited availability of proteins of the major light-harvesting complex of PSII caused by the chaos mutation. Immunoblot and pigment content analyses confirm that steadystate levels of Lhcb1, Lhcb2, Lhcb3, and the minor antenna Lhcb6 are markedly reduced in chaos and prors $1-1$ chaos mutants (this manuscript; Klimyuk et al., 1999). The observation that transcripts of the plastid $p s a A-B$ operon are less abundant in chaos and prors1-1 chaos leaves, but higher in prors1-1 chloroplasts, than in WT supports the idea that the PQ pool is more oxidized 

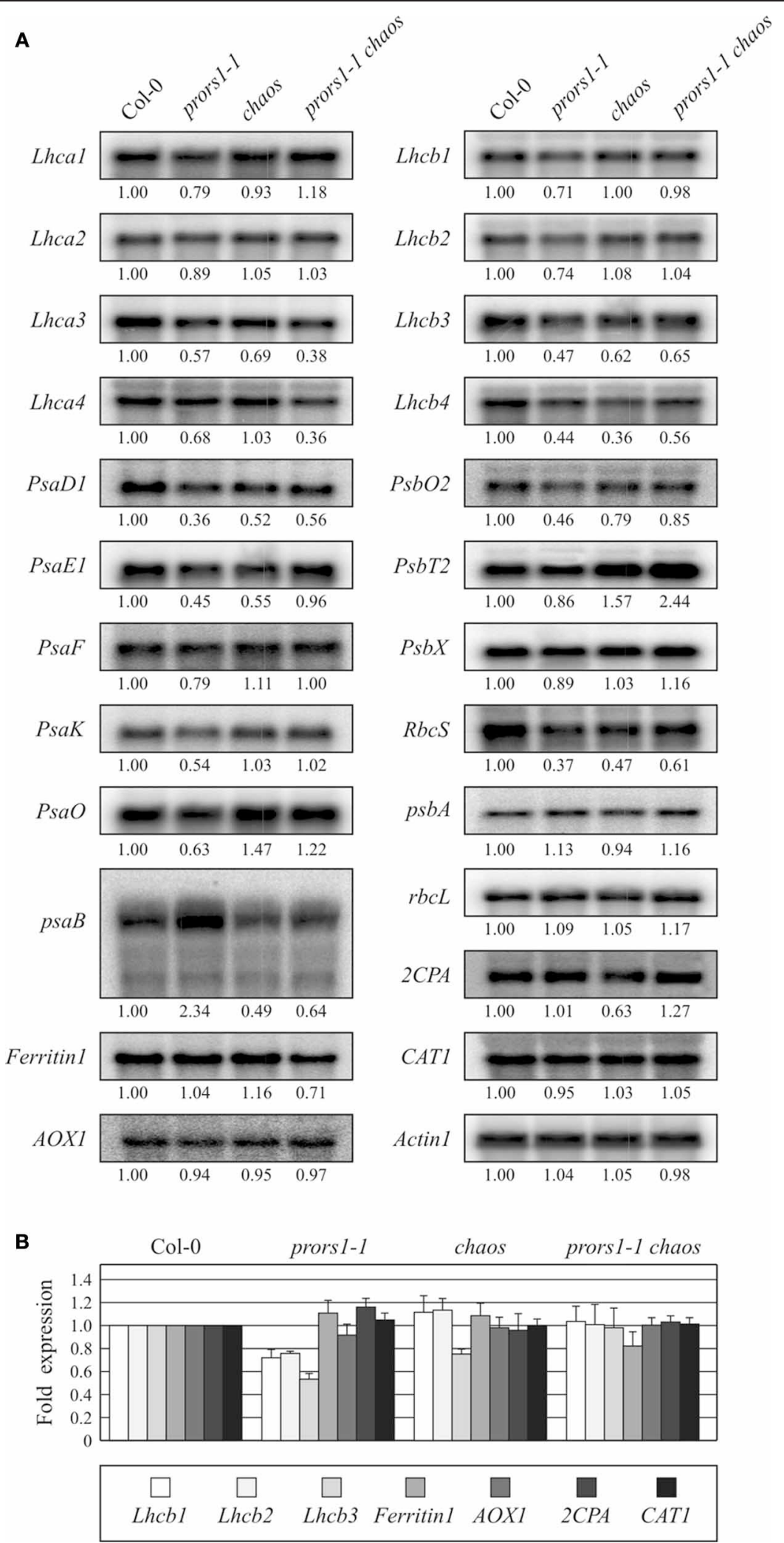

FIGURE 5 | Expression analysis of photosynthesis and antioxidant genes in WT (Col-0) and mutant (prors 1-1, chaos, and prors 1-1 chaos) leaves.

(A) Twenty microgram samples of total RNA from light-adapted WT, prors 1-1, chaos, and prors 1-1 chaos mutant plants were size-fractionated by agarose gel electrophoresis, transferred to nitrocellulose filters and probed with cDNA fragments specific for transcripts encoding subunits of PSI (Lhca1, -2, -3, -4, psaB, PsaD1, PsaE1, PsaF, PsaK, PsaO), PSII (Lhcb1, -2,-3,-4, psbA, PsbO2, (Continued) 


\section{FIGURE 5 | Continued}

$P s b T 2, P s b X)$ and Rubisco (RbcS, RbcL). Expression of nuclear genes encoding antioxidant enzymes, such as Ferritin1, AOX1, 2CPA, CAT1, and $A O X 1$, was also monitored. Three independent RNA gel-blot analyses were performed for each gene. Equal loading of RNA was checked by

hybridization with Actin1 specific probe. (B) Expression of genes belonging to multi-gene families, such as Lhcb1, Lhcb2, Lhcb3, Ferritin1, AOX1, 2CPA, and CAT1, was also monitored by qRT-PCR as described in "Materials and Methods." Average values from three biological and three technical replicates are reported. Note that the expression level of each of the gene analyzed was normalized to 1 in Col-0 background. Bars indicate standard deviations. in the presence of the chaos mutation, because expression of this operon is promoted by a reduced PQ pool (Pfannschmidt et al., 1999a,b; Allen and Pfannschmidt, 2000). Despite the partial reversal of the hyper-reduction of the thylakoid electron transport chain, the prors 1-1 chaos plants showed a reduction of about $50 \%$ in growth rate relative to prors $1-1$ single mutants under growth chamber conditions, most probably as a result of the constraints on $\mathrm{O}_{2}$ evolution, and consequently on ATP and NADPH production.

\section{LIGHT ABSORPTION AND THE INFLUENCE OF TRS ON THE OGE-DEPENDENT RETROGRADE PATHWAY}

The alleviation of the hyper-reduction of the thylakoid electron transport chain caused by the prors1-1 mutation, together with the persistence of the defect in protein synthesis in plastids and mitochondria, as demonstrated by in vivo labeling assays (see Figure 4), make prors1-1 chaos plants ideal material for distinguishing between OGE- and TRS-dependent retrograde signals. Analyses of nuclear genes which are down-regulated in prors1-1 (this manuscript; Pesaresi et al., 2006), and form part of a dynamic inter-compartmental transcriptional network dedicated to adjusting the activity of organelles in response to the cellular energy state and environmental stress (Biehl et al., 2005; Leister et al., 2011), support the notion that TRS and OGE play important roles in retrograde signaling. The prors1-1 chaos plants show a general derepression of nuclear photosynthetic genes relative to the prors1-1 mutant. More specifically, the genes affected could be divided into four major groups: (1) genes transcribed at higher levels than in WT (like PsbT2), (2) genes transcribed at WT levels (Lhca1, Lhca2, PsaE1, PsaF, PsaK, $P s a O, L h c b 1, L h c b 2$, and $P s b X)$, (3) genes expressed at levels higher than in prors1-1 but lower than in WT leaves (PsaD1, Lhcb3, Lhcb4, PsbO2, and RbcS), and (4) genes whose expression was not derepressed by the chaos mutation (Lhca3 and Lhca4). Hence, in the prors1-1 chaos double mutant, the decrease in the TRS stimulus (caused by the chaos mutation) neutralizes some of the gene regulatory effects of the prors1-1 mutation. This observation allows us to conclude that these genes are regulated by TRS (and possibly also indirectly by the chloroplast redox state and the associated carbon metabolism), or synergistically by TRS and OGE. Indeed, we would argue that TRS and OGE signals together contribute to retrograde signaling, although the degree to which their action is synergistic varies from gene to gene. Interestingly, the transcriptional derepression effect observed in prors1-1 chaos leaves is associated with increased accumulation of most of the corresponding gene products, indicating the physiological significance of this regulation of transcript abundance. Thus, with the major exception of proteins whose accumulation requires their cpSRP43-mediated, post-translational insertion into thylakoids (i.e., Lhcb subunits), the levels of other photosynthetic proteins analyzed were generally higher in the prors1-1 chaos double mutant than in prors1-1, and essentially identical to those seen in WT (see Figure 2 and Table 3).

However, it should not be forgotten that the down-regulation of nuclear photosynthesis genes in prors1-1 leaves persists even after dark adaptation, which would suggest that the OGEdependent retrograde pathway is largely independent of light, and thus of photosynthesis and TRS (Pesaresi et al., 2006). However, this is more likely to be an example of "systemic acquired acclimation" (SAA), where plants retain a "memory" of stress conditions induced by environmental or, as in the case of prors1-1, genetic factors, which allows them to mount a more effective defence against further episodes of such a stress (Karpinski et al., in press). The phenomenon is triggered by systemic redox changes in the thylakoid electron transport chain, and it avoids the need for prors 1-1 plants to induce the response to excess excitation energy de novo every time a dark-to-light transition takes place, thus saving energy for other metabolic pathways.

Because changes in OGE inevitably affect TRS and may also result in ROS-mediated oxidative stress, TRS and/or ROS might contribute directly to OGE signaling. However, the fact that there is no change in the expression of genes involved in detoxifying ROS, such as Ferritin1, 2CPA, CAT1 and AOX1, argues against the possibility that the OGE signaling pathway is triggered by oxidative stress in prors 1-1 plants (this manuscript; Pesaresi et al., 2006). Nonetheless, an involvement of ROS, in particular of $\mathrm{H}_{2} \mathrm{O}_{2}$, in OGE signaling cannot be entirely ruled out, since studies of isolated thylakoid membranes and intact chloroplasts have shown that a fraction of the plastid-produced $\mathrm{H}_{2} \mathrm{O}_{2}$ reaches the cytosol even under low-light conditions, which argues that physiological levels of $\mathrm{H}_{2} \mathrm{O}_{2}$ may play a role in signaling (Bienert et al., 2007; Mubarakshina et al., 2010).

On the other hand, changes in photosynthetic electron transport and the associated alteration of the redox state of PQ pool and stromal compounds per se, cannot explain the coordinated down-regulation of nuclear photosynthesis gene expression observed in prors 1-1 leaves. This is because only the simultaneous impairment of mitochondrial and plastid OGE results in the down-regulation of most of the nuclear photosynthetic genes in this genotype (Pesaresi et al., 2006). Indeed, lightshift experiments in combination with DCMU treatments have demonstrated that only 54 nuclear Arabidopsis genes are under the direct control of the PQ redox state, and only two of these codes for components directly associated with photosynthesis (Fey et al., 2005). Moreover, the derepression of photosynthesis gene expression in prors1-1 chaos leaves is only partial (the accumulation of Lhca3, Lhca4, PsaD1, Lhcb3, Lhcb4, PsbO2, and 
$R b c S$ transcripts was not restored to WT levels), implying the existence of multiple signal sources in both plastids and mitochondria, which must be integrated to enable the re-orchestration of photosynthetic NGE.

Since, photosynthetic electron transport is responsible of synthesis of carbohydrates that, in turn, are consumed in mitochondria by respiration, sugars may be also regarded as signaling metabolites involved in retrograde communication (Baier and Dietz, 2005). Thus, increased levels of glucose or sucrose repress photosynthesis gene expression, by involving hexokinase that is critical for sensing and responding to hexose signals, intracellularly (Jang et al., 1997; Rolland et al., 2006). Moreover, ABA has been also proposed to play a role in retrograde signaling, due to the fact that ABA biosynthesis starts into the chloroplasts and that $\mathrm{ABA}$ is strictly interconnected with photosynthesis (Baier et al.,

\section{REFERENCES}

Allen, J. F., and Pfannschmidt, T. (2000). Balancing the two photosystems: photosynthetic electron transfer governs transcription of reaction centre genes in chloroplasts. Philos. Trans. R. Soc. Lond. B Biol. Sci. 355, 1351-1357.

Amin, P., Sy, D. A., Pilgrim, M. L., Parry, D. H., Nussaume, L., and Hoffman, N. E. (1999). Arabidopsis mutants lacking the 43- and 54kilodalton subunits of the chloroplast signal recognition particle have distinct phenotypes. Plant Physiol. 121, 61-70.

Apel, K., and Hirt, H. (2004). Reactive oxygen species: metabolism, oxidative stress, and signal transduction. Annu. Rev. Plant Biol. 55, 373-399.

Baier, M., and Dietz, K. J. (2005). Chloroplasts as source and target of cellular redox regulation: a discussion on chloroplast redox signals in the context of plant physiology. J. Exp. Bot. 56, 1449-1462.

Baier, M., Stroher, E., and Dietz, K. J. (2004). The acceptor availability at photosystem I and ABA control nuclear expression of 2Cys peroxiredoxin-A in Arabidopsis thaliana. Plant Cell Physiol. 45, 997-1006.

Barajas-Lopez, J. D., Blanco, N. E., and Strand, A. (in press). Plastidto-nucleus communication, signals controlling the running of the plant cell. Biochim. Biophys. Acta. doi: 10.1016/j.bbr.2011.03.031

Bassi, R., Peruffo, A. D., Barbato, R., and Ghisi, R. (1985). Differences in chlorophyll-protein complexes and composition of polypeptides between thylakoids from bundle sheaths and mesophyll-cells in maize. Eur. J. Biochem. 146, 589-595.

Biehl, A., Richly, E., Noutsos, C., Salamini, F., and Leister, D. (2005).
Analysis of 101 nuclear transcriptomes reveals 23 distinct regulons and their relationship to metabolism, chromosomal gene distribution and co-ordination of nuclear and plastid gene expression. Gene 344, 33-41.

Bienert, G. P., Moller, A. L., Kristiansen, K. A., Schulz, A., Moller, I. M., Schjoerring, J. K., et al. (2007). Specific aquaporins facilitate the diffusion of hydrogen peroxide across membranes. Eur. J. Biochem. $282,1183-1192$.

Estavillo, G. M., Crisp, P. A., Pornsiriwong, W., Wirtz, M., Collinge, D., Carrie, C., et al. (2011). Evidence for a SAL1-PAP chloroplast retrograde pathway that functions in drought and high light signaling in Arabidopsis. Plant Cell 23, 3992-4012.

Farber, A., Young, A. J., Ruban, A. V., Horton, P., and Jahns, P. (1997). Dynamics of xanthophyll-cycle activity in different antenna subcomplexes in the photosynthetic membranes of higher plants (the relationship between zeaxanthin conversion and nonphotochemical fluorescence quenching). Plant Physiol. 115, 1609-1618.

Fey, V., Wagner, R., Brautigam, K., Wirtz, M., Hell, R., Dietzmann, A., et al. (2005). Retrograde plastid redox signals in the expression of nuclear genes for chloroplast proteins of Arabidopsis thaliana. J. Biol. Chem. 280, 5318-5328.

Giraud, E., Ho, L. H., Clifton, R., Carroll, A., Estavillo, G., Tan, Y. F., et al. (2008). The absence of ALTERNATIVE OXIDASEla in Arabidopsis results in acute sensitivity to combined light and drought stress. Plant Physiol. 147, 595-610.

2004). As a matter of fact, recent findings have placed the transcription factor ABI4 at the crossroads between mitochondrial and chloroplast retrograde signaling pathways and perhaps as a convergence point for mitochondria-plastid-nucleus coordination (Giraud et al., 2008). Nevertheless, prors1-1 plants appear to be able to maintain a memory of stress conditions, suggesting that also chromatin remodeling factors, and not only transcription factors, may have a prominent role in retrograde signaling.

\section{ACKNOWLEDGMENTS}

This work was supported by grants to Paolo Pesaresi from the Italian Ministry of Research (Special Fund for Basic Research, Grant No. PRIN 2008XB7774B) and to Dario Leister from the Deutsche Forschungsgemeinschaft (SFB-TR1, TP B8, and FOR 804). We thank Paul Hardy for critical reading of the manuscript.

Havaux, M., and Devaud, A. (1994). Photoinhibition of photosynthesis in chilled potato leaves is not correlated with a loss of photosystem-II activity - preferential inactivation of photosystem-I. Photosynth. Res. 40, 75-92.

Ihnatowicz, A., Pesaresi, P., Varotto, C. Richly, E., Schneider, A., Jahns, P. et al. (2004). Mutants for photosystem I subunit D of Arabidopsis thaliana: effects on photosynthesis, photosystem I stability and expression of nuclear genes for chloroplast functions. Plant J. 37, 839-852.

Jang, J. C., Leon, P., Zhou, L., and Sheen, J. (1997). Hexokinase as a sugar sensor in higher plants. Plant Cell 9, 5-19.

Jung, H. S., Okegawa, Y., Shih, P. M., Kellogg, E., Abdel-Ghany, S. E., Pilon, M., et al. (2010). Arabidopsis thaliana PGR7 encodes a conserved chloroplast protein that is necessary for efficient photosynthetic electron transport. PloS ONE 5:e11688. doi: 10.1371/journal.pone.0011688

Karpinski, S., Szechynska-Hebda, M. Wituszynska, W., and Burdiak, P. (in press). Light acclimation, retrograde signalling, cell death, and immune defences in plants. Plant Cell Environ. doi: 10.1111/pce.12018

Keegstra, K., and Cline, K. (1999). Protein import and routing systems of chloroplasts. Plant Cell 11, 557-570.

Kleine, T., Maier, U. G., and Leister, D. (2009a). DNA transfer from organelles to the nucleus: the idiosyncratic genetics of endosymbiosis. Annu. Rev. Plant Biol. 60, 115-138.

Kleine, T., Voigt, C., and Leister, D. (2009b). Plastid signalling to the nucleus: messengers still lost in the mists? Trends Genet. 25, 185-192.
Klenell, M., Morita, S., Tiemblo-Olmo, M., Muhlenbock, P., Karpinski, S., and Karpinska, B. (2005). Involvement of the chloroplast signal recognition particle cpSRP43 in acclimation to conditions promoting photooxidative stress in Arabidopsis. Plant Cell Physiol. 46, 118-129.

Klimyuk, V. I., Persello-Cartieaux, F. Havaux, M., Contard-David, P., Schuenemann, D., Meiherhoff, K., et al. (1999). A chromodomain protein encoded by the Arabidopsis $C A O$ gene is a plant-specific component of the chloroplast signal recognition particle pathway that is involved in LHCP targeting. Plant Cell 11, 87-99.

Koussevitzky, S., Nott, A., Mockler, T. C., Hong, F., Sachetto-Martins, G., Surpin, M., et al. (2007). Signals from chloroplasts converge to regulate nuclear gene expression. Science 316, 715-719.

Kramer, D. M., Johnson, G., Kiirats, O., and Edwards, G. E. (2004). New fluorescence parameters for the determination of $\mathrm{Q}_{\mathrm{A}}$ redox state and excitation energy fluxes. Photosynth. Res. 79, 209-218.

Leister, D. (2005). Origin, evolution and genetic effects of nuclear insertions of organelle DNA. Trends Genet. 21, 655-663.

Leister, D. (2012). Retrograde signaling in plants: from simple to complex scenarios. Front. Plant Sci. 3:135. doi: 10.3389/fpls.2012.00135

Leister, D., Varotto, C., Pesaresi, P., Niwergall, A., and Salamini, F. (1999). Large-scale evaluation of plant growth in Arabidopsis thaliana by non-invasive image analysis. Plant Physiol. Biochem. 37, 671-678.

Leister, D., Wang, X., Haberer, G., Mayer, K. F., and Kleine, T. (2011) 
Intracompartmental and intercompartmental transcriptional networks coordinate the expression of genes for organellar functions. Plant Physiol. 157, 386-404.

Martinez-Garcia, J. F., Monte, E., and Quail, P. H. (1999). A simple, rapid and quantitative method for preparing Arabidopsis protein extracts for immunoblot analysis. Plant J. 20, 251-257.

Mateo, A., Muhlenbock, P., Rusterucci, C., Chang, C. C., Miszalski, Z., Karpinska, B., et al. (2004). LESION SIMULATING DISEASE 1 is required for acclimation to conditions that promote excess excitation energy. Plant Physiol. 136, 2818-2830.

Mochizuki, N., Tanaka, R., Tanaka, A., Masuda, T., and Nagatani, A. (2008). The steady-state level of Mgprotoporphyrin IX is not a determinant of plastid-to-nucleus signaling in Arabidopsis. Proc. Natl. Acad. Sci. U.S.A. 105, 15184-15189.

Moller, I. M., and Sweetlove, L. J. (2010). ROS signalling-specificity is required. Trends Plant Sci. 15, 370-374.

Moulin, M., McCormac, A. C., Terry, M. J., and Smith, A. G. (2008). Tetrapyrrole profiling in Arabidopsis seedlings reveals that retrograde plastid nuclear signaling is not due to Mg-protoporphyrin IX accumulation. Proc. Natl. Acad. Sci. U.S.A. 105, 15178-15183.

Mubarakshina, M. M., Ivanov, B. N., Naydov, I. A., Hillier, W., Badger, M. R., and Krieger-Liszkay, A. (2010). Production and diffusion of chloroplastic $\mathrm{H}_{2} \mathrm{O}_{2}$ and its implication to signalling. J. Exp. Bot. 61, 3577-3587.

Oelze, M. L., Kandlbinder, A., and Dietz, K. J. (2008). Redox regulation and overreduction control in the photosynthesizing cell: complexity in redox regulatory networks. Biochim. Biophys. Acta 1780, 1261-1272.

Pesaresi, P. (2011). Studying translation in Arabidopsis chloroplasts. Methods Mol. Biol. 774, 209-224.
Pesaresi, P., Hertle, A., Pribil, M., Kleine, T., Wagner, R., Strissel, H., et al. (2009). Arabidopsis STN7 kinase provides a link between short- and long-term photosynthetic acclimation. Plant Cell 21, 2402-2423.

Pesaresi, P., Masiero, S., Eubel, H., Braun, H. P., Bhushan, S., Glaser, E., et al. (2006). Nuclear photosynthetic gene expression is synergistically modulated by rates of protein synthesis in chloroplasts and mitochondria. Plant Cell 18, 970-991.

Pesaresi, P., Schneider, A., Kleine, T., and Leister, D. (2007). Interorganellar communication. Curr. Opin. Plant Biol. 10, 600-606.

Pesaresi, P., Varotto, C., Meurer, J., Jahns, P., Salamini, F., and Leister, D. (2001). Knock-out of the plastid ribosomal protein L11 in Arabidopsis: effects on mRNA translation and photosynthesis. Plant J. 27, 179-189.

Pfannschmidt, T., Nilsson, A., and Allen, J. F. (1999a). Photosynthetic control of chloroplast gene expression. Nature 397, 625-628.

Pfannschmidt, T., Nilsson, A., Tullberg, A., Link, G., and Allen, J. F. (1999b). Direct transcriptional control of the chloroplast genes $p s b A$ and $p s a A B$ adjusts photosynthesis to light energy distribution in plants. IUBMB Life 48, 271-276.

Pfannschmidt, T., and Yang, C. (2012). The hidden function of photosynthesis: a sensing system for environmental conditions that regulates plant acclimation responses. Protoplasma 249 (Suppl. 2), 125-136.

Pogson, B. J., Woo, N. S., Forster, B., and Small, I. D. (2008). Plastid signalling to the nucleus and beyond. Trends Plant Sci. 13, 602-609.

Ramel, F., Birtic, S., Ginies, C., Soubigou-Taconnat, L., Triantaphylides, C., and Havaux, M. (2012). Carotenoid oxidation products are stress signals that mediate gene responses to singlet oxygen in plants. Proc. Natl. Acad. Sci. U.S.A. 109, 5535-5540.
Rolland, F., Baena-Gonzalez, E., and Sheen, J. (2006). Sugar sensing and signaling in plants: conserved and novel mechanisms. Annu. Rev. Plant Biol. 57, 675-709.

Sambrook, J., and Russell, D. W. (2001). Molecular Cloning: A Laboratory Manual. 3rd Edn. Cold Spring Harbor, NY: Cold Spring Harbor Laboratory Press.

Schägger, H., and von Jagow, G. (1987). Tricine-sodium dodecyl sulfatepolyacrylamide gel electrophoresis for the separation of proteins in the range from 1 to $100 \mathrm{kDa}$. Anal. Biochem. 166, 368-379.

Schuenemann, D., Gupta, S., PerselloCartieaux, F., Klimyuk, V. I., Jones, J. D., Nussaume, L., et al. (1998). A novel signal recognition particle targets light-harvesting proteins to the thylakoid membranes. Proc. Natl. Acad. Sci. U.S.A. 95, 10312-10316.

Strand, A., Asami, T., Alonso, J., Ecker, J. R., and Chory, J. (2003). Chloroplast to nucleus communication triggered by accumulation of Mg-protoporphyrinIX. Nature 421, 79-83.

Sullivan, J. A., and Gray, J. C. (1999). Plastid translation is required for the expression of nuclear photosynthesis genes in the dark and in roots of the pea lip1 mutant. Plant Cell 11, 901-910.

Sun, X., Feng, P., Xu, X., Guo, H., Ma, J., Chi, W., et al. (2011). A chloroplast envelope-bound PHD transcription factor mediates chloroplast signals to the nucleus. Nat. Commun. 2:477. doi: 10.1038/ncomms1486

Varotto, C., Pesaresi, P., Maiwald, D., Kurth, J., Salamini, F., and Leister, D. (2000). Identification of photosynthetic mutants of Arabidopsis by automatic screening for altered effective quantum yield of photosystem 2. Photosynthetica 38, 497-504.

Wisman, E., Hartmann, U., Sagasser, M., Baumann, E., Palme, K., Hahlbrock, K., et al. (1998). Knock-out mutants from an En-1 mutagenized Arabidopsis thaliana population generate phenylpropanoid biosynthesis phenotypes. Proc. Natl. Acad. Sci. U.S.A. 95, 12432-12437.

Woodson, J. D., and Chory, J. (2008). Coordination of gene expression between organellar and nuclear genomes. Nat. Rev. Genet. 9, 383-395.

Woodson, J. D., Perez-Ruiz, J. M., and Chory, J. (2011). Heme synthesis by plastid ferrochelatase I regulates nuclear gene expression in plants. Curr. Biol. 21, 897-903.

Xiao, Y., Savchenko, T., Baidoo, E. E., Chehab, W. E., Hayden, D. M., Tolstikov, V., et al. (2012). Retrograde signaling by the plastidial metabolite MEcPP regulates expression of nuclear stress-response genes. Cell 149, 1525-1535.

Conflict of Interest Statement: The authors declare that the research was conducted in the absence of any commercial or financial relationships that could be construed as a potential conflict of interest.

Received: 24 September 2012; accepted: 30 November 2012; published online: 27 December 2012.

Citation: Tadini L, Romani I, Pribil $M$, Jahns $P$, Leister $D$ and Pesaresi $P$ (2012) Thylakoid redox signals are integrated into organellar-gene-expressiondependent retrograde signaling in the prors1-1 mutant. Front. Plant Sci. 3:282. doi: $10.3389 /$ fpls.2012.00282

This article was submitted to Frontiers in Plant Physiology, a specialty of Frontiers in Plant Science.

Copyright (C) 2012 Tadini, Romani, Pribil, Jahns, Leister and Pesaresi. This is an open-access article distributed under the terms of the Creative Commons Attribution License, which permits use, distribution and reproduction in other forums, provided the original authors and source are credited and subject to any copyright notices concerning any thirdparty graphics etc. 